\title{
Cellular apoptosis susceptibility (CAS) is overexpressed in thyroid carcinoma and maintains tumor cell growth: A potential link to the $B R A F^{\mathrm{V} 600 \mathrm{E}}$ mutation
}

\author{
KERSTIN HOLZER ${ }^{1}$, ELISABETH DRUCKER ${ }^{1}$, SCOTT OLIVER $^{1}$, JULIANE WINKLER ${ }^{1}$, \\ EVA EITENEUER $^{1}$, ESTHER HERPEL $^{1,2}$, KAI BREUHAHN ${ }^{1}$ and STEPHAN SINGER ${ }^{1}$ \\ ${ }^{1}$ Institute of Pathology, University Hospital Heidelberg; \\ ${ }^{2}$ Tissue Bank of the National Center for Tumor Diseases (NCT) Heidelberg, 69120 Heidelberg, Germany
}

Received December 7, 2015; Accepted January 18, 2016

DOI: 10.3892/ijo.2016.3388

\begin{abstract}
Thyroid carcinoma is among the most common malignant endocrine neoplasms with a rising incidence. Genetic alterations occurring in thyroid cancer frequently affect the RAS/RAF/MEK/ERK-pathway such as the oncogenic, kinase-activating $\mathrm{BRAF}^{\mathrm{V} 600 \mathrm{E}}$ mutation. Nuclear transport receptors including importins and exportins represent an important part of the nuclear transport machinery providing nucleo-cytoplasmic exchange of macromolecules. The role of nuclear transport receptors in the development and progression of thyroid carcinomas is largely unknown. Here, we studied the expression and function of the exportin cellular apoptosis susceptibility (CAS) in thyroid carcinogenesis and its link to the $\mathrm{BRAF}^{\mathrm{V} 600 \mathrm{E}}$ mutation. By using immunohistochemistry (IHC) we found significantly increased IHC scores of CAS in primary papillary (PTC) and medullary (MTC), but not in follicular (FTC) thyroid carcinoma compared to non-tumorous (NT) thyroid tissue. Interestingly, metastases of the aforementioned subtypes including FTC showed a strong CAS positivity. Among PTCs we observed that CAS immunoreactivity was significantly higher in the tumors harboring the $\mathrm{BRAF}^{\mathrm{V} 600 \mathrm{E}}$ mutation. Furthermore, depletion of CAS by RNAi in the BRAF ${ }^{\mathrm{V} 600 \mathrm{E}}$-positive PTC cell line B-CPAP led to reduced tumor cell growth measured by crystal violet assays. This phenotype could be attributed to reduced proliferation and increased cell death as assayed by BrdU ELISAs and immunoblotting for PARP-cleavage, respectively. Finally, we found additive effects of CAS siRNA and vemurafenib treatment in B-CPAP cells. Collectively, these data suggest that CAS
\end{abstract}

Correspondence to: Dr Stephan Singer, Institute of Pathology, University Hospital Heidelberg, Im Neuenheimer Feld 224, 69120 Heidelberg, Germany

E-mail: stephan.singer@med.uni-heidelberg.de

Key words: thyroid cancer, cellular apoptosis susceptibility, vemurafenib, BRAF, nuclear transport overexpression in thyroid carcinoma depends on the subtype and the disease stage. Our findings also indicate that CAS maintains PTC cell proliferation and survival. Targeting CAS could represent a potential therapeutic approach particularly in combination with BRAF inhibitors such as vemurafenib in $\mathrm{BRAF}^{\mathrm{V} 600 \mathrm{E}}$-positive tumors.

\section{Introduction}

Thyroid cancer is among the most common endocrine malignancies with a rising incidence over the last decades (1). The most common variants of primary malignant thyroid neoplasms comprise papillary thyroid carcinoma (PTC) and follicular thyroid carcinoma (FTC) that are both derived from the follicular epithelium. In contrast, medullary thyroid cancer (MTC) that arises from parafollicular C-cells accounts only for a minor proportion of thyroid malignancies (1). Among frequently activated oncogenic signaling pathways in thyroid cancer is the RAS/RAF/MEK/ERK (MAPK) pathway (1). The MAPK pathway transduces signals from the extracellular environment through the cytoplasm to the nucleus and activates a variety of biochemical processes such as cell proliferation, differentiation, and apoptosis $(2,3)$. Extracellular stimuli include growth factors, hormones, and cytokines interacting with their respective receptors which (via adaptor proteins) activate small guanine nucleotidebinding proteins belonging to the Ras superfamily of small GTPases. Ras in turn recruits and activates RAF proteins such as the kinase BRAF (V-raf murine sarcoma viral oncogene homolog B1) to initiate subsequent phosphorylation events including MEK1/2 and ERK1/2 to activate downstream transcription factors mediating the aforementioned cellular outcomes $(2,3)$. Frequent genetic alterations in thyroid cancer that result in aberrant activation of the RAS/RAF/MEK/ERK pathway include the RAS genes $H R A S, K R A S$ and NRAS as well as $B R A F$, found in $61.7 \%$ of PTCs mostly represented by the $B R A F^{V 600 E}$ mutation (4) leading to a single amino acid substitution from valine to glutamic acid at codon 600 (5). Oncogenic $B R A F^{V 600 E}$ was reported to be associated with a poor clinical outcome, recurrence and treatment failure in PTC (6-8). Vemurafenib is a potent kinase inhibitor of 
$\mathrm{BRAF}^{\mathrm{V} 600 \mathrm{E}}$ which is successfully used in patients with malignant melanoma $(5,9)$ and may also have beneficial effects in patients with metastatic PTC (10).

The nuclear transport machinery is essential to eukaryotic cell function by providing the selective exchange of macromolecules between the nucleus and the cytoplasm (11). Nuclear transport receptors represent an important part of the nuclear transport system and belong mainly to the karyopherin superfamily (11). This includes importins, exportins, and transportins shuttling cargos between the nuclear and cytoplasmic compartment through the nuclear pore complex in the respective direction $(12,13)$. The exportin cellular apoptosis susceptibility (CAS) is a pivotal transport factor by re-shuttling importin- $\alpha$ (imp- $\alpha$ ) from the nucleus to the cytoplasm for subsequent importin- $\beta 1$ (imp- $\beta 1$ )/imp- $\alpha$-dependent import of protein cargos $(12,14)$. As indicated by its name CAS was first described and characterized as an apoptosis susceptibility protein by Brinkmann et al $(15,16)$. Moreover, CAS was reported to be overexpressed in a variety of solid tumors [breast $(17)$, liver $(18,19)$ and ovarian $(20)$ cancer] suggesting also pro-tumorigenic properties. However, the expression profile and functional role of CAS in thyroid carcinoma also in the context of the frequently occurring driver mutation $B R A F^{V 600 E}$ has not been reported so far.

Here, we demonstrate that CAS expression depends on the histological subtype, the disease stage and on the presence or absence of the $B R A F^{V 600 E}$ mutation. Our data also suggest the requirement of CAS to maintain cell growth and survival in a $B R A F^{V 600 E}$ expressing PTC cell line as well as additive effects of CAS siRNA and vemurafenib treatment.

\section{Material and methods}

Tissue microarray (TMA). The TMA of thyroid carcinomas contained 49 tumor samples and 17 non-tumor (NT) thyroid tissue samples from patients who underwent surgery at the University Hospital Heidelberg or at other local hospitals between 2002 and 2011. It includes 17 NT, 14 FTC, 20 PTC and 15 MTC largely represented by two cores per patient except for small sized tumors due to limited tumor extent. The tumors were diagnosed based on the latest classification of endocrine tumors of the World Health Organization (2004). Tissue samples were provided by the tissue bank of the National Center for Tumor Diseases (NCT, Heidelberg, Germany) in accordance with the regulations of the tissue bank and the approval of the Ethics Committee of Heidelberg University. Tumor tissues were formalin fixed, paraffin-embedded and slides were stained with hematoxylin and eosin (H\&E). These full-section $H \& E$ slides were re-evaluated by two independent pathologists assigning representative and viable tumor areas for coring. The TMA block was cut with a microtome into $5-\mu \mathrm{m}$ sections, which were mounted on glass slides and processed for immunohistochemical staining.

The TMA of lymph node and soft tissue metastasis of different thyroid carcinomas contained 5 lymph node metastases of PTCs, 6 soft tissue metastases and 1 lung metastasis of FTCs and 4 lymph node metastases and 2 lung metastases of MTCs. With a tissue microarray instrument (Beecher MTA-1; Beecher Instruments, Inc., Sun Prairie, WI, USA) $2 \mathrm{~mm}$-diameter cores were punched from the assigned areas of the donor blocks and arrayed on the recipient (TMA) block for further processing as described above.

Immunohistochemical staining (IHC) procedure and evaluation. Immunohistochemical staining was performed with a commercially available monoclonal anti-CAS mouse antihuman antibody (Ab54674; Abcam, Cambridge, UK) in a 1:50 dilution (Dako REAL Antibody Diluent; Dako, Hamburg, Germany) based on a well-established protocol as previously described (19).

$\mathrm{BRAF}^{\mathrm{V} 600 \mathrm{E}}$ immunohistochemical staining was performed with a commercially available ready-to-use monoclonal mouse anti-human antibody (790-4855, 12,0 $\mu \mathrm{g} / \mathrm{ml}$; F. Hoffmann-La Roche AG, Basel, Switzerland) by using an automated immunostaining instrument (BenchMark Ultra IHC/ISH staining module; Ventana Medical Systems, Tucson, AZ, USA). Based on the manufacturer's protocol the OptiView DAB IHC Detection kit (OptiView; Ventana Medical Systems) was used. The procedure included the following steps: 4 min deparaffination at $62^{\circ} \mathrm{C}$, rinsing with EZ Prep, incubation with Cell Conditioner No. 1 (both from Ventana Medical Systems) for $64 \mathrm{~min}$ at $90^{\circ} \mathrm{C}, 24 \mathrm{~min}$ treatment with the primary antibody at $36^{\circ} \mathrm{C}, 4 \mathrm{~min}$ exposure to OptiView Peroxidase Inhibitor, 12 min incubation with OptiView HQ Universal Linker, 12 min treatment in OptiView HRP Multimer, 8 min incubation with a mixture of OptiView $\mathrm{H}_{2} \mathrm{O}_{2}$ and DAB, 4 min exposure to OptiView copper, counterstaining with haematoxylin for $12 \mathrm{~min}, 4 \mathrm{~min}$ incubation with bluing reagent. Each incubation was followed by multiple rinsing steps in reaction buffer (Ventana Medical Systems). Dehydration of each TMA slide was performed as follows: 1x5 min 70\% ethanol, 1x5 min $96 \%$ ethanol, $2 \times 5$ min $100 \%$ ethanol, 1x5 min Xylene by using the Leica Autostainer XL. Finally the slides were mounted with cover slips (Leica CV5030).

The immunohistochemical staining of cytoplasmic and nuclear CAS was semi-quantitatively evaluated using a score calculated by multiplying staining intensity and quantity. Intensity range: 0 , negative; 1 , weak; 2 , moderate; and 3 , strong. Quantity range: 0 , no staining; 1 , staining in $<1 \% ; 2$, staining in $<10 \%$; 3 , staining in $10-50 \%$; and 4 , staining in $>50 \%$ of tumor cells. In case of 2 representative areas of tumor samples the mean of the two resulting products was used.

Cell culture. The papillary thyroid cancer cell line B-CPAP was cultured in Roswell Park Memorial Institute (RPMI)-1640 medium with $10 \%$ fetal calf serum and $1 \%$ penicillin/streptomycin. RPMI media and the antibiotics were purchased from Sigma (Munich, Germany).

Transfection of siRNAs. CAS-specific siRNAs were purchased from Eurofins MWG Operon (Ebersberg, Germany), transfected with Oligofectamine (Invitrogen, Karlsruhe, Germany) according to the manufacturer's instructions and used at a final concentration of $50 \mathrm{nM}$. Sequences were as follows CAS\#1, 5'-CUGACGGUAUCAAAUAUAUUA-3', CAS\#2, 5'-GGAA CUCAGCGAUGCAAAU-3'. The Qiagen All-Stars duplex served as the negative control for all knockdown experiments.

Cell growth assays. Cells were seeded into 96-well plates $24 \mathrm{~h}$ after siRNA transfection. The cell number of each 
A

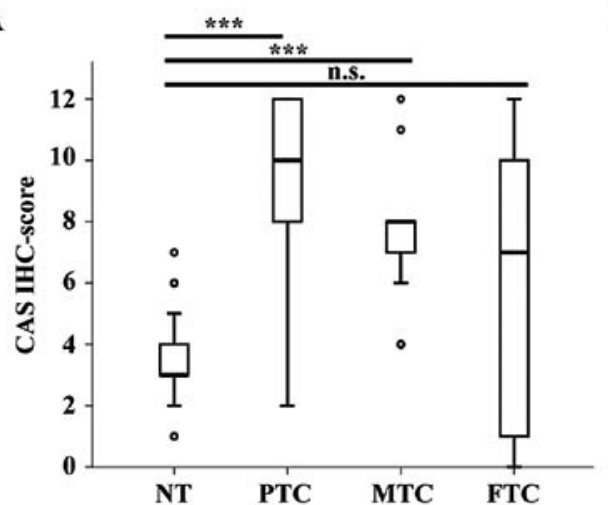

C

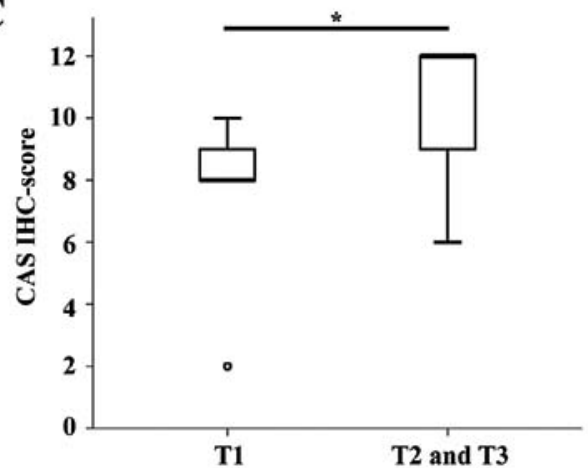

B
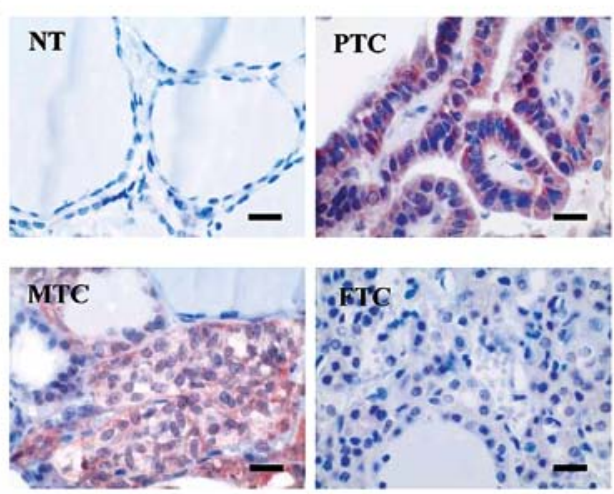

Figure 1. CAS is overexpressed in primary PTC and MTC. (A) Boxplot shows median semiquantitative immunohistochemical (IHC) scores (cytoplasmic) for non-tumorous thyroid tissue (NT, n=17), papillary thyroid carcinoma (PTC, n=20), medullary thyroid carcinoma (MTC, $\mathrm{n}=15$ ) and follicular thyroid carcinoma (FTC, $n=14$ ). Asterisks indicate significance level ${ }^{* * * *}$ p $<0.001$. (B) IHC of CAS in representative tissue specimen of NT, PTC, MTC and FTC. Scale bar, $20 \mu \mathrm{m}$. (C) Boxplot shows median semiquantitative immunohistochemical (IHC) scores (cytoplasmic) for different tumor stages in PTC. Asterisk indicates significance level ${ }^{*} \mathrm{p}<0.05$.

condition was assessed at different time-points after transfection by using crystal-violet (Serva, Heidelberg, Germany) as a staining reagent. Dried cells were solubilized in 50\% ethanolic 1 M Natriumcitrate solution and colorimetric measurement was performed at $550 \mathrm{~nm}$ using an ELISA reader (FLUOstar Omega; BMG Labtech, Ortenberg, Germany) and normalized to the control siRNA condition. Cell proliferation was examined using a bromodeoxyuridine enzyme-linked immunosorbent assay (Cell Proliferation Biotrak ELISA system, version 2; GE Healthcare/Amersham, Freiburg, Germany) according to the manufacturer's instructions and the optical density was measured at $450 \mathrm{~nm}$ using a microplate photometer (Multiskan Ascent; Thermo Electron Corporation, Waltham, MA, USA).

Gel electrophoresis and immunoblotting. Cells were harvested in cell lysis buffer (Cell Signaling/New England Biolabs, Frankfurt, Germany) supplemented with a protease inhibitor cocktail (Serva, Heidelberg, Germany). The concentration of whole cell protein lysates was determined by Bradford assays (Bio-Rad Protein assay; Bio-Rad, Hercules, CA, USA). After boiling the samples for $5 \mathrm{~min}$ at $90^{\circ} \mathrm{C}$, proteins were separated by SDS/PAGE and transferred to a nitrocellulose membrane (Whatman, Dassel, Germany). The membrane was blocked with 5\% milk/TBST (Milchpulver; Roth, Karlsruhe, Germany) for $1 \mathrm{~h}$ and incubated overnight with anti-CAS antibody (Ab54674; Abcam, Cambridge, UK) diluted 1:500, with anti- $\beta$-actin antibody (MP Biomedicals,
Illkirch, France) diluted 1:10,000, anti-pERK antibody (9101S) diluted 1:500 and anti-PARP antibody (9542L; both from Cell Signaling Technology) in a 1:500 dilution. After rinsing with TBST the membranes where incubated for $1 \mathrm{~h}$ with the corresponding fluorescently-labeled secondary antibodies (LI-COR Bioscience, Bad Homburg, Germany). After washing with TBST signal detection was performed using Odysee Sa Infrared Imaging system (LI-COR Bioscience).

\section{Results}

We first set out to analyze the expression and intracellular localization of CAS in different subtypes of thyroid carcinoma compared to NT thyroid tissue. To do so, we performed immunohistochemical CAS staining of a TMA consisting of 20 PTC, 14 FTCs, 15 MTC, and 17 NT. The patient characteristics are listed in Table I. The median IHC score (calculated by multiplying staining intensity and quantity) was significantly higher in PTCs and MTCs compared to NT (Fig. 1A). In contrast, CAS immunostaining was not significantly different between FTCs and NT. Representative pictures of each tumor subtype are displayed in Fig. 1B and illustrate that CAS was predominantly localized in the cytoplasm. These data suggest that CAS is overexpressed in primary PTCs and MTCs, but not in primary FTCs compared to NT. In addition, among primary PTCs we found significantly higher IHC scores of CAS in advanced T-stages (pT2 and pT3) compared to the early T-stage (pT1), as demonstrated in Fig. 1C. 
Table I. Patient characteristics including age, gender, pT-stage, and metastases.

\begin{tabular}{lcccc}
\hline Patient characteristics & Non-tumor $(\mathrm{n}=17)$ & PTC $(\mathrm{n}=20)$ & MTC $(\mathrm{n}=15)$ & FTC $(\mathrm{n}=14)$ \\
\hline Age (years) & & & & $62 \pm 20$ \\
Mean \pm SD & $50 \pm 17$ & $50 \pm 15$ & $15-82$ & $29-87$ \\
Range & $15-87$ & $25-83$ & & 9 \\
Gender & & & 9 & 5 \\
Female & 10 & 16 & 6 & 2 \\
Male & 7 & 4 & 10 & 2 \\
Stage & - & 5 & 2 & 9 \\
T1 & - & 6 & 3 & 1 \\
T2 & - & 9 & - & 1 \\
T3 & - & - & & 7 \\
T4 & - & 5 & 2 & \\
Metastases & - & - & & \\
Lymph node & - & & & \\
Distant & & & & \\
\hline
\end{tabular}

While the aforementioned analyses were performed on primary tumors we investigated CAS expression in lymph node (N) or hematogenous [distant (D)] metastases of PTCs, MTCs and FTCs. Interestingly, regardless of the subtype the large majority of metastatic tumors exhibited a strong CAS cytoplasmic immunoreactivity reflected by a maximum IHC score of 12 (Fig. 2A). In addition, most of the metastatic neoplasms also displayed a moderate to strong nuclear CAS positivity (Fig. 2B) that was rarely observed in the primary tumors. The comparison of those carcinomas for which the primary tumor and the metastases of the same patient were available indicates that CAS immunostaining was either increased in the metastasis or maintained on a high level (Fig. 2C and D), but never decreased. We concluded that upon metastatic spread thyroid carcinomas including FTC are characterized by a strong CAS expression with cytoplasmic and nuclear localization.

Among the most frequent driver mutations in PTC is the BRAF $^{\mathrm{V} 600 \mathrm{E}}$ mutation (4). Thus, we tested if CAS IHC scores differ between $\mathrm{BRAF}^{\mathrm{V} 600 \mathrm{E}}$ positive and negative tumors as classified by a specific BRAF ${ }^{\mathrm{V} 600 \mathrm{E}}$ antibody. In fact, CAS immunoreactivity was significantly higher in $\mathrm{BRAF}^{\mathrm{V} 600 \mathrm{E}}$ positive PTCs (median IHC score of 11 vs. 7) (Fig. 3A). Representative pictures of CAS and BRAF ${ }^{\mathrm{V} 600 \mathrm{E}}$ IHCs of the respective PTC groups are shown in Fig. 3B.

Based on these findings we chose the PTC cell line $\mathrm{B}-\mathrm{CPAP}$ reported to express the $\mathrm{BRAF}^{\mathrm{V} 600 \mathrm{E}}$ mutation for functional characterization of CAS in vitro by using RNA $i$. We first tested the knockdown efficiency of two different CAS-specific siRNAs (CAS\#1 and CAS\#2) by immunoblotting (Fig. 4A) and densitometric analyses (Fig. 4B). Fig. 4A and B demonstrate up to $90 \%$ reduction of CAS protein compared to the control siRNA (ctrl.) treated condition. Encouraged by this high knockdown efficiency we then determined how CAS depletion affects tumor cell growth in B-CPAP by monitoring the cell number of each knockdown condition 48,96 and $144 \mathrm{~h}$ after transfection using crystal violet assays. Fig. $4 \mathrm{C}$ shows that the number of CAS siRNA-treated cells was strikingly lower with up to $70 \%$ less cells after $144 \mathrm{~h}$ of transfection compared to the controls. We further investigated if this decrease in tumor cell number was caused by reduced proliferation and/or increased cell death. The proliferation activity in the presence or absence of CAS was measured by BrdU assays. Indeed, upon CAS depletion BrdU incorporation was significantly reduced compared to the control siRNA treated cells (Fig. 4D) indicating less proliferative capacity. Furthermore, CAS knockdown was also associated with increased apoptosis as indicated by elevated PARP cleavage (Fig. 4E).

Taken together, these data suggest that CAS is essential for tumor cell growth in PTC by maintaining cell proliferation and preventing cell death.

Considering the immunohistochemical profile and the function of CAS we hypothesized that CAS could be downstream of the MAPK signaling cascade. We therefore determined how treatment of B-CPAP cells with the BRAF inhibitor vemurafenib affects CAS protein levels. Interestingly, vemurafenib treatment for 24 and $48 \mathrm{~h}$ at a concentration of 1 and $10 \mu \mathrm{M}$ did not affect CAS protein (Fig. 5A), while it was sufficient to downregulate pERK (Fig. 5A) and to reduce cell growth (Fig. 5B). This suggested that vemurafenib and CAS siRNA treatment reduce cell growth independently. Therefore, we tested if combining both treatments leads to additive effects. Indeed, even a low-dose treatment of vemurafenib of $0.5 \mu \mathrm{M}$ that itself had only a mild effect on tumor cell viability, resulted in a further reduction of tumor cell number in the combined treatment compared to the CAS-siRNA treatment alone (Fig. 5C). Collectively, these data suggest that targeting CAS and BRAF in BRAF ${ }^{\mathrm{V} 600 \mathrm{E}}$ positive PTCs could be a promising therapeutic approach.

\section{Discussion}

In the present study we analyzed the immunohistochemical profile of the nuclear transport factor CAS in primary and 
A

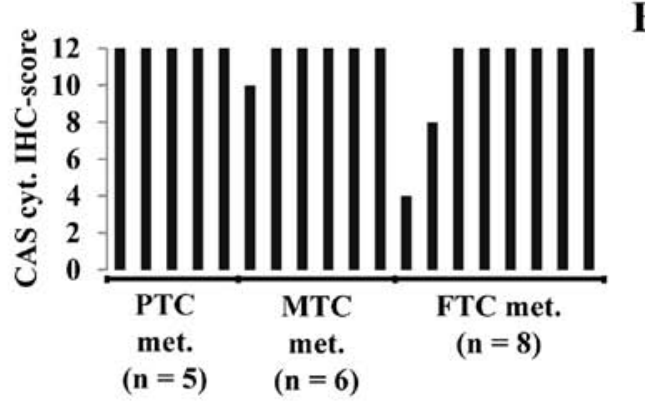

B

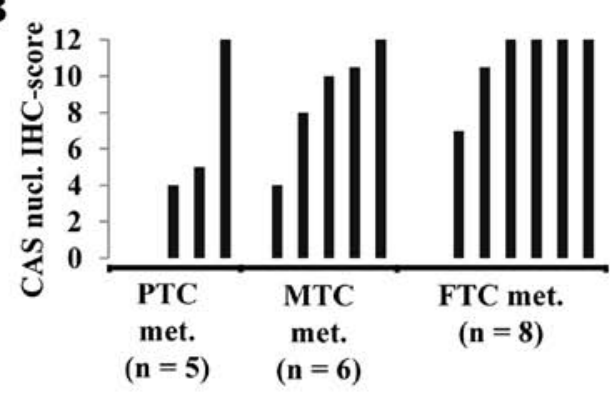

C

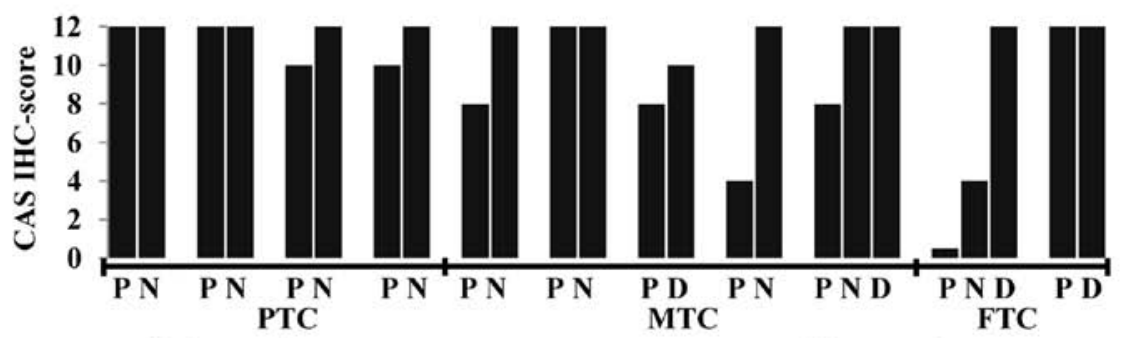

D
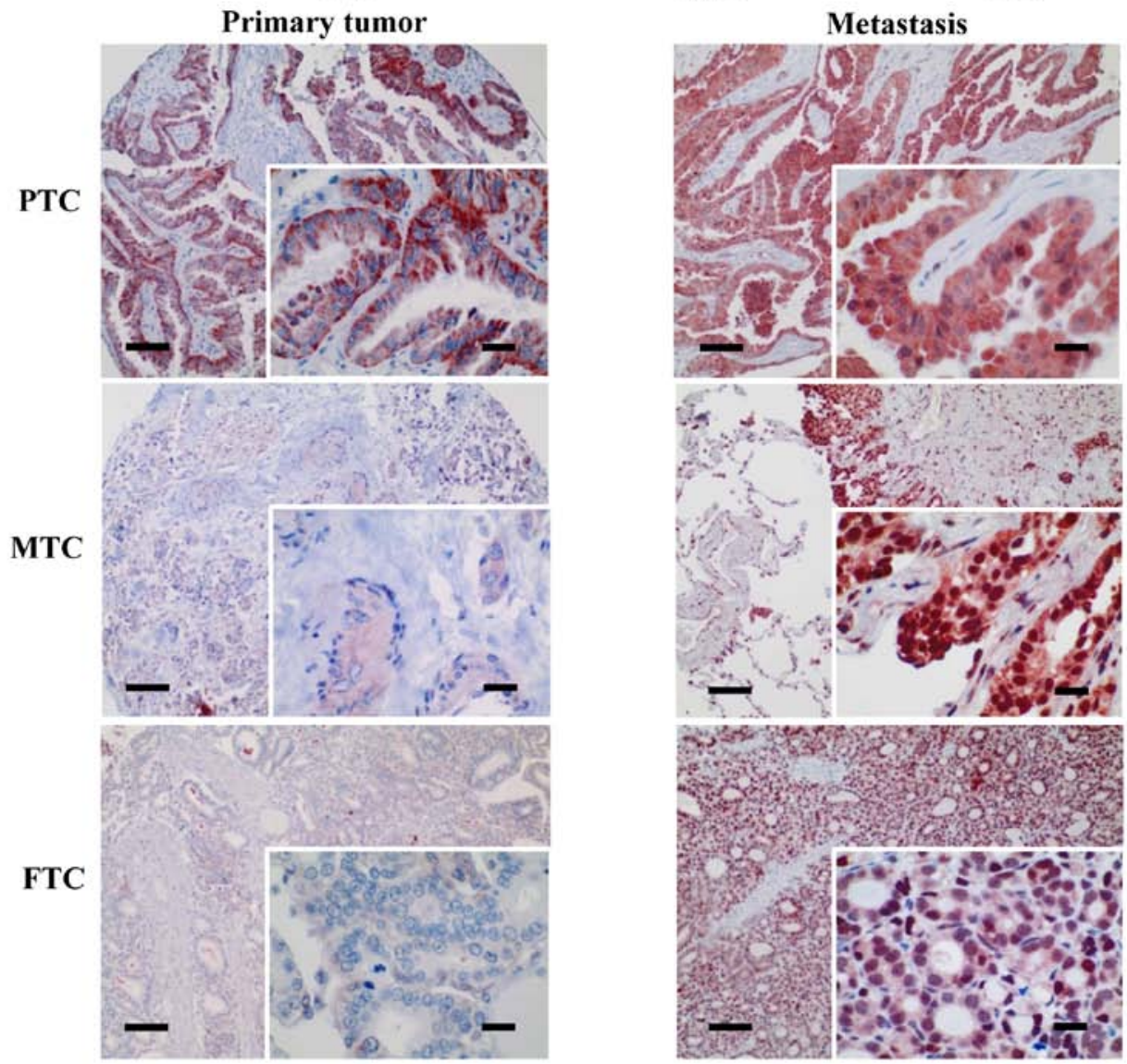

Figure 2. CAS is highly expressed in metastatic PTC, MTC, and FTC. Bar diagram indicates the cytoplasmic (A) or nuclear (B) semiquantitative CAS immunohistochemical (IHC) score for each metastatic (met.) PTC, MTC and FTC. (C) Bar diagram indicates cytoplasmic CAS IHC scores for corresponding primary tumor $(\mathrm{P})$, lymph node $(\mathrm{N})$ or distant (D) metastasis. (D) IHC of CAS in representative tissue specimen of corresponding primary and metastatic PTC (upper row), MTC (middle row), and FTC (lower row). Scale bar $20 \mu \mathrm{m}$, (inset $100 \mu \mathrm{m}$ ).

metastatic thyroid carcinomas. Including different carcinoma subtypes such as PTC, FTC and MTC in the analyses revealed that overexpression of CAS appears to be subtype-dependent at least in the primary tumors. However, the fact that CAS immunoreactivity was significantly higher only in primary PTC and MTC, but not in primary FTC needs further validation in larger patient cohorts before firm conclusions can be drawn. Nevertheless, increased CAS expression in PTC and
MTC is in principle consistent with previous studies describing high expression levels of CAS in other carcinomas such as liver (18,19), breast (17), ovarian (20), endometrial (21) and prostate (22) cancer, among others. The immunohistological CAS data are also consistent with the functional experiments upon CAS depletion indicating that CAS is required to maintain cell growth of thyroid cancer cells. The BrdU assays in B-CPAP indicate that the reduced cell number after CAS knockdown 
A

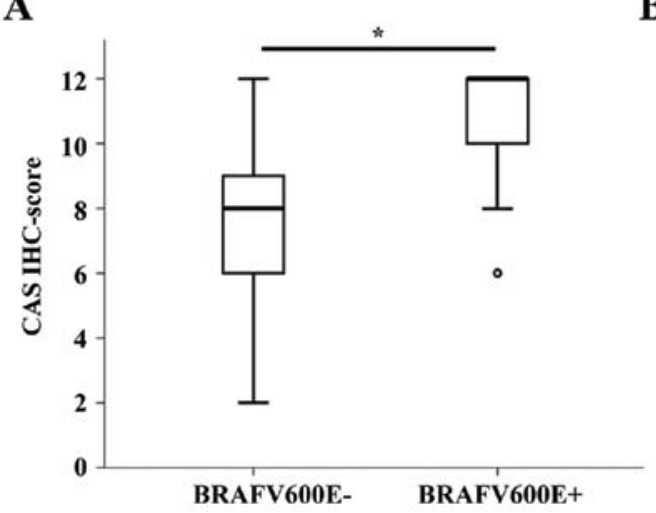

B

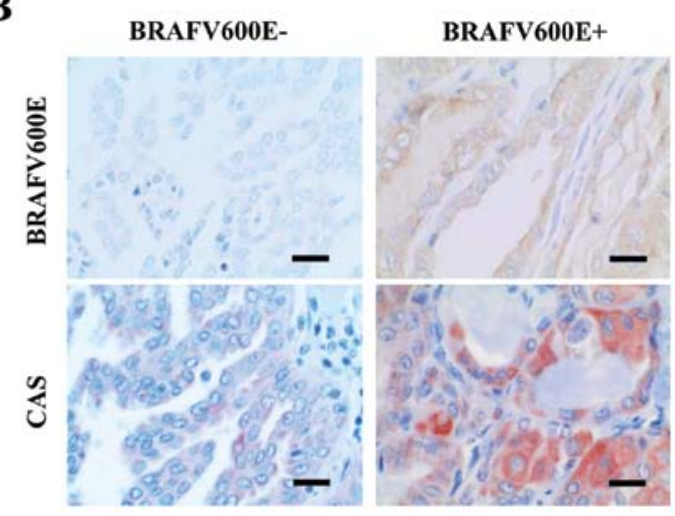

Figure 3. CAS is higher expressed in PTC containing the BRAF ${ }^{\mathrm{V} 600 \mathrm{E}}$ mutation. (A) Boxplot indicates the semiquantitative CAS immunohistochemical (IHC) scores for PTCs with and without the BRAF ${ }^{\mathrm{V} 600 \mathrm{E}}$ mutation. (B) $\mathrm{IHC}_{\text {of }} \mathrm{BRAF}^{\mathrm{V} 600 \mathrm{E}}$ (upper row) and CAS (lower row) in representative tissue specimen of the indicated PTC groups. Scale bar, $20 \mu \mathrm{m}$.

A

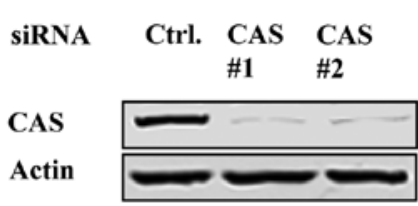

C

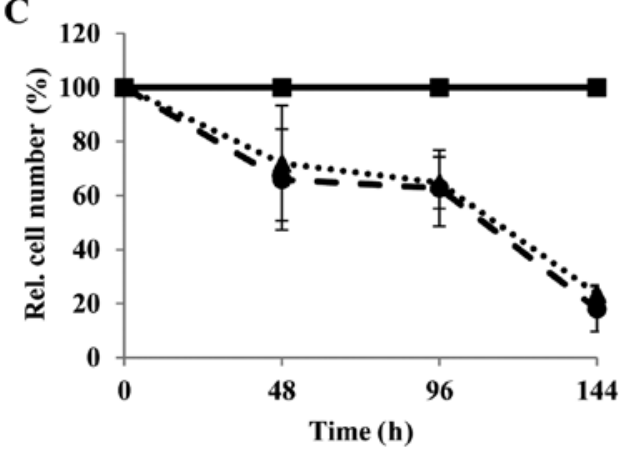

D

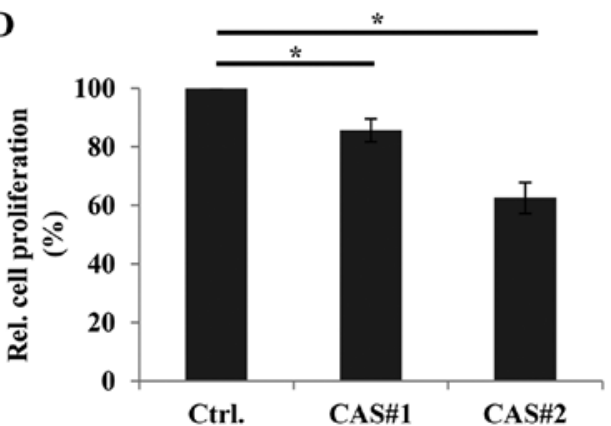

B

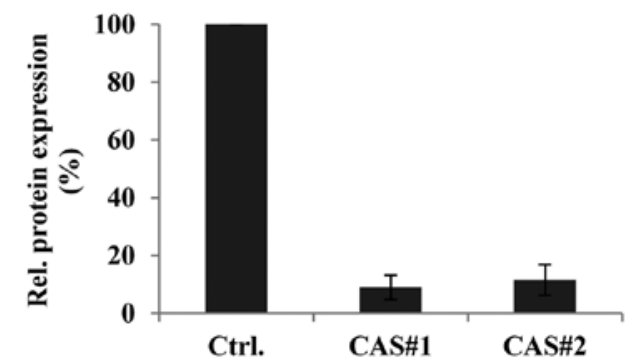

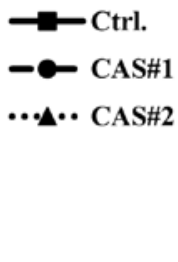

$\mathbf{E}$

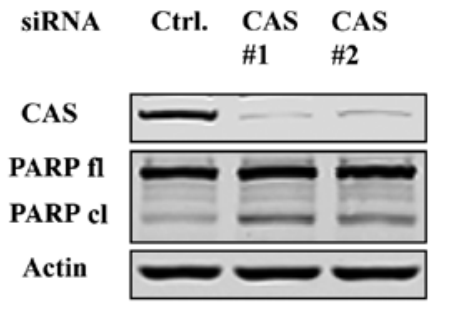

Figure 4. CAS is required for tumor cell growth in B-CPAP. (A) B-CPAP cells were treated either with a control siRNA (ctrl.) or two CAS-specific siRNAs (CAS\#1 and CAS\#2) and cell extracts were immunoblotted with the indicated antibodies. (B) Relative CAS protein expression of the indicated conditions derived from densitometric analyses of three biological replicates. (C) Relative number of cells (\%) normalized to the control siRNA condition at each time-point was analyzed 48, 96 and $144 \mathrm{~h}$ after transfection using crystal violet assays. Data are presented as the mean \pm SD derived from three biological replicates. CAS depletion reduces proliferation and increases cell death in B-CPAP cells. (D) B-CPAP cells were treated either with a control siRNA (ctrl.) or CAS-specific siRNA (CAS\#1 and CAS\#2). Relative BrdU incorporation (\%) normalized to the control siRNA condition was assayed three days after siRNAtreatment for each condition. Data are presented as the mean $\pm \mathrm{SD}$ derived from three biological replicates ${ }^{*} \mathrm{p}<0.05$. (E) B-CPAP cells were treated either with a control siRNA or two CAS-specific siRNAs (CAS\#1 and CAS\#2) and cell extracts were immunoblotted with the indicated antibodies.

compared to the control siRNA treated condition is at least in part the result of reduced cell proliferation. Several studies reported that high expression levels of CAS can be found in high proliferating tissues and tumors $(17,18,23,24)$. Nevertheless, 
$\mathbf{A}$

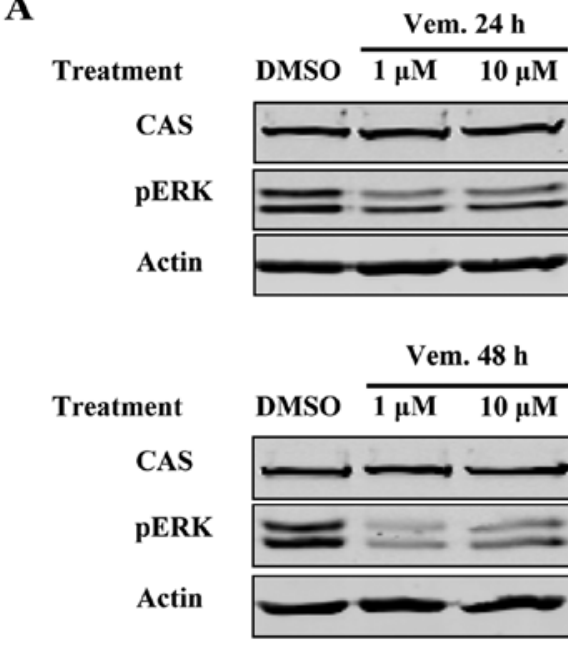

B

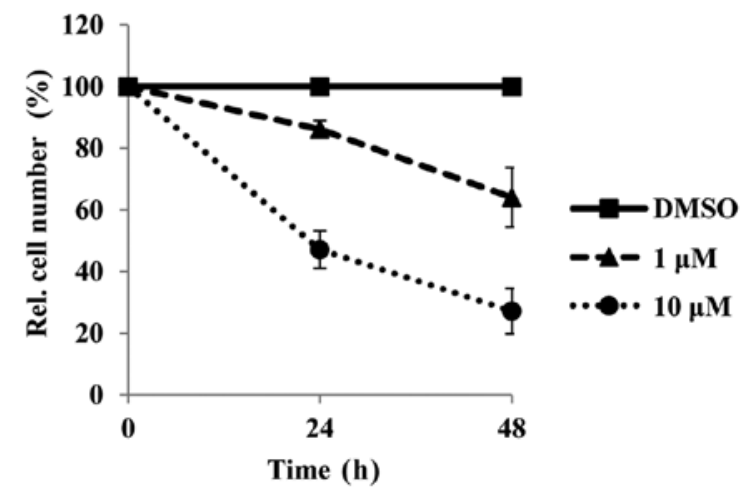

C

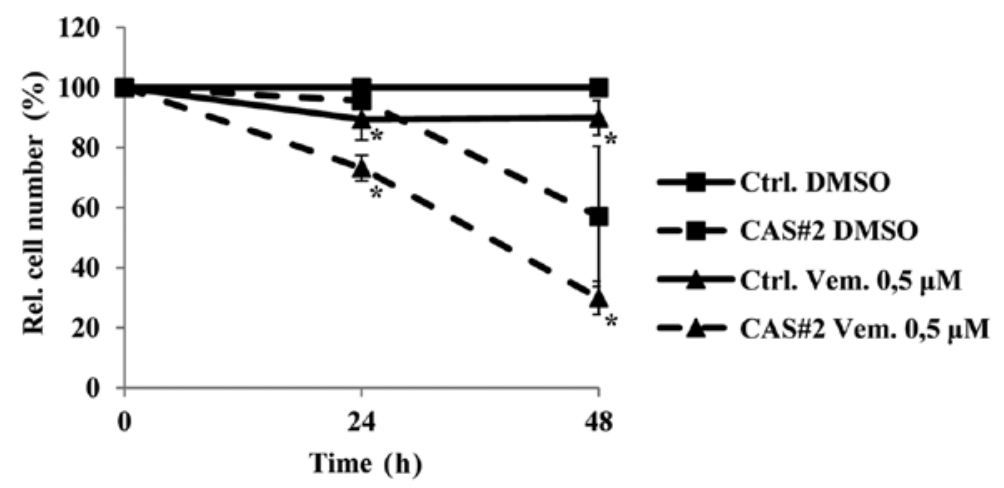

Figure 5. Additive effects of vemurafenib and CAS siRNA treatment in B-CPAP cells. (A) B-CPAP cells were treated with 1 or $10 \mu \mathrm{M}$ vemurafenib for 24 (upper panel) and $48 \mathrm{~h}$ (lower panel). Cell extracts were immunoblotted using the indicated antibodies. (B) B-CPAP cells were treated with the same concentrations of vemurafenib as described in (A). Relative number of cells (\%) normalized to the DMSO condition at each time-point was analyzed 24 and $48 \mathrm{~h}$ after adding vemurafenib using crystal violet assays. (C) B-CPAP cells were treated either with a control siRNA (ctrl.) or two CAS-specific siRNAs (CAS\#1 and CAS\#2). Forty-eight hours after transfection DMSO (D) or vemurafenib (V) was added for additional 24 or $48 \mathrm{~h}$. Relative number of cells (\%) normalized to the ctrl siRNA + DMSO condition was analyzed by crystal violet assays. Data are presented as the mean \pm SD derived from three biological replicates * $<<0.05$.

considering CAS as a proliferation-associated protein remains a matter of debate in the field (25-27). Exogenous CAS overexpression from a cDNA-construct in HT-29 (colon carcinoma) and MCF-7 (breast cancer) failed to increase, and instead reduced cell proliferation $(26,27)$. Therefore, the authors concluded that the role of CAS in cancer progression is not stimulating proliferation. Further studies predominantly from the same group rather emphasize a role of CAS in migration, invasion, and metastatic spread $(25,26,28,29)$. The latter would also be in line with the expression pattern of CAS described in this study that was particularly high in metastatic thyroid carcinomas. Besides reduced cell proliferation we also found induced PARP-cleavage in cells depleted of CAS which indicates an increase in apoptotic cell death. This finding is consistent with previous analyses in HCC cells (19), where CAS depletion resulted in higher Caspase 3-activity and increased PARP-cleavage. However, these observations need to be discussed in light of the initially described function of CAS as a pro-apoptotic factor $(15,16)$. Differences in the experimental set up and cell line variability require consideration in this matter. Brinkmann et al $(15,16)$ conducted their experiments under stress-conditions (TNF $\alpha$ - and exotoxin-treatment), while we studied CAS function under regular, non-stress conditions. Here we used the thyroid carcinoma cell line B-CPAP as opposed to MCF-7 cells analyzed in the aforementioned study. Finally, the timeframe of the analyses seems a decisive determinant as well. For instance, in another study focusing on CAS in the context of p53-mediated apoptosis Tanaka et al (30) report reduced apoptosis in MCF-7 cells after UV-treatment for $36 \mathrm{~h}$ in the CAS-knockdown conditions, but subsequent cell death at later time-points. Clearly, these data demonstrate that CAS plays a dual and context-dependent role in apoptosis. To further explore the functions of CAS in thyroid cancer it will be interesting to investigate how papillary carcinoma cell lines with lower CAS expression or non-tumorigenic thyroid cells behave upon CAS overexpression. Titration of a CAS cDNA expression construct in time course experiments may reveal if pro- or anti-apoptotic/-proliferative functions are determined by dosage and timing in this particular tumor entity.

The $B R A F^{V 600 E}$ mutation has received wide attention as an important factor correlated to an aggressive course of PTC and as a predictor for disease recurrence $(7,8)$. However, some concerns have been raised regarding its prognostic and predictive power, which may be explained by a recent study showing that BRAF ${ }^{\mathrm{V} 600 \mathrm{E}} \mathrm{PTC}$ s consist of at least four molecular subtypes (4). We demonstrate that $\mathrm{BRAF}^{\mathrm{V} 600 \mathrm{E}_{-}}$ positive PTCs show significantly higher CAS IHC scores compared to those being negative for this mutation, but it 
remains to be investigated in a larger patient cohort if and how the aforementioned molecular subtypes differ in their CAS immunoreactivity and if CAS itself is of prognostic value.

Surprising in this context was the finding that CAS protein remained unaltered in B-CPAP cells even after high-dose treatment of vemurafenib, since the IHC analysis suggested that CAS could be a downstream target of the RAS/RAF/MEK/ERK pathway. That vemurafenib was effective in these cells could be demonstrated by reduced ERK $1 / 2$ phosphorylation and reduced cell number in the treatment condition. This raises the possibility that either the residual amount of phosphorylated ERK was still sufficient to maintain CAS expression or that another pathway can compensate the vemurafenib mediated BRAF blockade. Transcription factors (TFs) possibly involved in the former scenario (as downstream targets of ERK) are Ets and Elk1. In fact, several binding sites of these TFs can be identified in the promoter region (as defined by 2000 bps upstream of the transcription start site) of CAS in silico (http://genome.ufl.edu/mapper/mapper-main). However, to further substantiate these assumptions chromatin immunoprecipitation experiments and luciferase assays would be required. Moreover, it is also conceivable that CAS may not be a direct downstream target of the MAPK signaling cascade at all, but may be required for preferentially used transport pathways in $\mathrm{BRAF}^{\mathrm{V} 600 \mathrm{E}}$ positive tumors. Elucidating the functional and regulatory link between $\mathrm{BRAF}^{\mathrm{V} 600 \mathrm{E}}$ and $\mathrm{CAS}$ represents a rewarding subject for future studies. Nonetheless, in light of apparently independent effects of CAS and $\mathrm{BRAF}^{\mathrm{V} 600 \mathrm{E}}$ on tumor cell viability the combinatorial treatment of CAS siRNA and vemurafenib could be the basis for a therapeutic approach. This, however, requires validation in several thyroid cancer cell lines and appropriate mouse models as well as testing to what extent 'normal', immortalized thyroid cells and nontumorous thyroid tissue are affected by this treatment. Once tumor-specific effects have been established it would also be of considerable interest to study if major effects of CAS on thyroid cancer cell growth and survival can be linked to its role as an exporter for importin- $\alpha$ s, such as imp- $\alpha 1$ [as previously shown for hepatocellular carcinoma (19)]. If so, a compound targeting the CAS/imp- $\alpha(1)$ interaction may be more effective than a CAS siRNA approach since the utility of siRNA therapeutics are limited by several factors (e.g. unfavorable physicochemical characteristics, instability with short plasma half-lives and lysosomal degradation upon endocytosis) (31).

Targeting a nuclear transport factor in a therapeutic context is not without precedence. For solid and hematological malignancies selective inhibitors of nuclear export (SINE), compounds inhibiting exportin-1 [chromosome region maintenance 1 (CRM1)] like selinexor, have already entered phase I/II of clinical trials (32). Future studies are required to show if CAS and other associated transport factors can serve as targets for effective molecular treatment strategies in thyroid carcinomas resistant to or recurrent upon conventional therapies.

\section{Acknowledgements}

We thank David Jansen and Veronika Geißler as well as other members of Tissue Bank of the National Center for Tumor Diseases (NCT) for their support. K.H. received a fellow- ship by the Rahel-Goitein-Straus Program, Medical Faculty Heidelberg. S.S. acknowledges funding by the German Research Foundation (Si1487/3-1).

\section{References}

1. Xing M: Molecular pathogenesis and mechanisms of thyroid cancer. Nat Rev Cancer 13: 184-199, 2013

2. Roberts PJ and Der CJ: Targeting the Raf-MEK-ERK mitogenactivated protein kinase cascade for the treatment of cancer. Oncogene 26: 3291-3310, 2007.

3. Cantwell-Dorris ER, O'Leary JJ and Sheils OM: BRAFV600E: Implications for carcinogenesis and molecular therapy. Mol Cancer Ther 10: 385-394, 2011.

4. Cancer Genome Atlas Research Network: Integrated genomic characterization of papillary thyroid carcinoma. Cell 159: 676-690, 2014.

5. Luke JJ and Hodi FS: Vemurafenib and BRAF inhibition: A new class of treatment for metastatic melanoma. Clin Cancer Res 18: 9-14, 2012.

6. Xing M, Westra WH, Tufano RP, Cohen Y, Rosenbaum E, Rhoden KJ, Carson KA, Vasko V, Larin A, Tallini G, et al: BRAF mutation predicts a poorer clinical prognosis for papillary thyroid cancer. J Clin Endocrinol Metab 90: 6373-6379, 2005.

7. Kim TH, Park YJ, Lim JA, Ahn HY, Lee EK, Lee YJ, Kim KW, Hahn SK, Youn YK, Kim KH, et al: The association of the BRAF(V600E) mutation with prognostic factors and poor clinical outcome in papillary thyroid cancer: A meta-analysis. Cancer 118: 1764-1773,2012.

8. Xing M, Alzahrani AS, Carson KA, Shong YK, Kim TY, Viola D, Elisei R, Bendlová B, Yip L, Mian C, et al: Association between BRAF V600E mutation and recurrence of papillary thyroid cancer. J Clin Oncol 33: 42-50, 2015.

9. Flaherty KT, Puzanov I, Kim KB, Ribas A, McArthur GA, Sosman JA, O'Dwyer PJ, Lee RJ, Grippo JF, Nolop K, et al: Inhibition of mutated, activated BRAF in metastatic melanoma. N Engl J Med 363: 809-819, 2010.

10. Kim KB, Cabanillas ME, Lazar AJ, Williams MD, Sanders DL, Ilagan JL, Nolop K, Lee RJ and Sherman SI: Clinical responses to vemurafenib in patients with metastatic papillary thyroid cancer harboring BRAF(V600E) mutation. Thyroid 23: 1277-1283, 2013.

11. Chook YM and Süel KE: Nuclear import by karyopherin- $\beta$ s: Recognition and inhibition. Biochim Biophys Acta 1813: 1593-1606, 2011.

12. Stewart M: Molecular mechanism of the nuclear protein import cycle. Nat Rev Mol Cell Biol 8: 195-208, 2007.

13. D'Angelo MA and Hetzer MW: Structure, dynamics and function of nuclear pore complexes. Trends Cell Biol 18: 456-466, 2008.

14. Kutay U, Bischoff FR, Kostka S, Kraft R and Görlich D: Export of importin alpha from the nucleus is mediated by a specific nuclear transport factor. Cell 90: 1061-1071, 1997.

15. Brinkmann U, Brinkmann E, Gallo M and Pastan I: Cloning and characterization of a cellular apoptosis susceptibility gene, the human homologue to the yeast chromosome segregation gene CSE1. Proc Natl Acad Sci USA 92: 10427-10431, 1995.

16. Brinkmann U, Brinkmann E, Gallo M, Scherf U and Pastan I: Role of CAS, a human homologue to the yeast chromosome segregation gene CSE1, in toxin and tumor necrosis factor mediated apoptosis. Biochemistry 35: 6891-6899, 1996.

17. Behrens P, Brinkmann U, Fogt F, Wernert N and Wellmann A: Implication of the proliferation and apoptosis associated CSE1L/CAS gene for breast cancer development. Anticancer Res 21: 2413-2417, 2001.

18. Wellmann A, Flemming P, Behrens P, Wuppermann K, Lang H, Oldhafer K, Pastan I and Brinkmann U: High expression of the proliferation and apoptosis associated CSE1L/CAS gene in hepatitis and liver neoplasms: Correlation with tumor progression. Int J Mol Med 7: 489-494, 2001.

19. Winkler J, Ori A, Holzer K, Sticht C, Dauch D, Eiteneuer EM, Pinna F, Geffers R, Ehemann V, Andres-Pons A, et al: Prosurvival function of the cellular apoptosis susceptibility/importin- $\alpha 1$ transport cycle is repressed by 553 in liver cancer. Hepatology 60: 884-895, 2014.

20. Brustmann H: Expression of cellular apoptosis susceptibility protein in serous ovarian carcinoma: A clinicopathologic and immunohistochemical study. Gynecol Oncol 92: 268-276, 2004. 
21. Peiró G, Diebold J, Baretton GB, Kimmig R and Löhrs U: Cellular apoptosis susceptibility gene expression in endometrial carcinoma: Correlation with Bcl-2, Bax, and caspase-3 expression and outcome. Int J Gynecol Pathol 20: 359-367, 2001.

22. Bar-Shira A, Pinthus JH, Rozovsky U, Goldstein M, Sellers WR, Yaron Y, Eshhar Z and Orr-Urtreger A: Multiple genes in human $20 \mathrm{q} 13$ chromosomal region are involved in an advanced prostate cancer xenograft. Cancer Res 62: 6803-6807, 2002.

23. Wellmann A, Krenacs L, Fest T, Scherf U, Pastan I, Raffeld M and Brinkmann U: Localization of the cell proliferation and apoptosis-associated CAS protein in lymphoid neoplasms. Am J Pathol 150: 25-30, 1997.

24. Böni R, Wellmann A, Man YG, Hofbauer G and Brinkmann U: Expression of the proliferation and apoptosis-associated CAS protein in benign and malignant cutaneous melanocytic lesions. Am J Dermatopathol 21: 125-128, 1999.

25. Tai CJ, Hsu CH, Shen SC, Lee WR and Jiang MC: Cellular apoptosis susceptibility (CSE1L/CAS) protein in cancer metastasis and chemotherapeutic drug-induced apoptosis. J Exp Clin Cancer Res 29: 110, 2010.

26. Liao CF, Luo SF, Li LT, Lin CY, Chen YC and Jiang MC: CSE1L/CAS, the cellular apoptosis susceptibility protein, enhances invasion and metastasis but not proliferation of cancer cells. J Exp Clin Cancer Res 27: 15, 2008.
27. Jiang MC and Liao CF: CSE1/CAS overexpression inhibits the tumorigenicity of HT-29 colon cancer cells. J Exp Clin Cancer Res 23: 325-332, 2004.

28. Yoshiura K, Nishishita T, Nakaoka T, Yamashita $\mathrm{N}$ and Yamashita N: Inhibition of B16 melanoma growth and metastasis in C57BL mice by vaccination with a syngeneic endothelial cell line. J Exp Clin Cancer Res 28: 13, 2009.

29. Tai CJ, Shen SC, Lee WR, Liao CF, Deng WP, Chiou HY, Hsieh CI, Tung JN, Chen CS, Chiou JF, et al: Increased cellular apoptosis susceptibility (CSE1L/CAS) protein expression promotes protrusion extension and enhances migration of MCF-7 breast cancer cells. Exp Cell Res 316: 2969-2981, 2010.

30. Tanaka T, Ohkubo S, Tatsuno I and Prives C: hCAS/CSE1L associates with chromatin and regulates expression of select p53 target genes. Cell 130: 638-650, 2007.

31. Wang J, Lu Z, Wientjes MG and Au JL: Delivery of siRNA therapeutics: Barriers and carriers. AAPS J 12: 492-503, 2010.

32. Senapedis WT, Baloglu E and Landesman Y: Clinical translation of nuclear export inhibitors in cancer. Semin Cancer Biol 27: 74-86, 2014. 